\title{
The hierarchical build-up of bulges in CDM
}

\author{
Sadegh Khochfar \\ Sub-Department of Astrophysics, University of Oxford, Denys Wilkinson Bldg., Keble Road, \\ OX1 3RH, Oxford, U.K. \\ email: sadeghk@astro.ox.ac.uk
}

\begin{abstract}
We investigate the hierarchical build-up of stars in bulges within the standard $\Lambda$ cold dark matter scenario. By separating the population into stars born during starbursts that accompany the formation of spheroids in major mergers (starburst component), and stars that are previously formed in discs of progenitor galaxies (quiescent component) and added to the spheroid by dynamical interaction. Our results are summarised as follows: bulges that form early have larger starburst fraction and hence should be smaller than their counter parts that form later. The quiescent fraction in bulges is an increasing function of bulge mass, becoming constant at $M_{\mathrm{q}} / M_{\mathrm{bul}} \sim 0.8$, mainly due to the infall of satellite galaxies that contribute disc stars to the bulge. Minor mergers are an order of magnitude more frequent than major mergers and must play a significant role in the evolution of bulges. Above the critical mass $M_{\mathrm{c}} \sim 3 \times 10^{10} \mathrm{M}_{\odot}$ most of the stars in the universe are in spheroids, which at high redshift are exclusively elliptical galaxies and at low redshifts partly bulges. Due to the enhanced evolution of galaxies ending up in high density environments, the starburst fraction and the surface mass densities of bulges below $M_{\mathrm{c}}$ should be enhanced with respect to field galaxies. Dissipation during the formation of massive bulges in present day early-type spirals is less important than for the formation of present day elliptical galaxies of the same mass thereby explaining the possible difference in phase-space densities between spiral galaxies and elliptical galaxies.
\end{abstract}

Keywords. Galaxies: bulges, galaxies: formation, galaxies: evolution, galaxies: interactions, galaxies: structure, methods: numerical

\section{Introduction}

The close resemblance of elliptical galaxies and classical bulges has lead to the widely accepted assumption that they have the same origin. Profiles of elliptical galaxies and bulges are nicely fit by Sersic-laws. The fact that super-massive black holes in bulges also follow the fundamental $M_{\bullet}-\sigma$-relation (Sarzi et al. 2001) provides further evidence for a common formation scenario of elliptical galaxies and classical bulges.

Early work by Toomre \& Toomre (1972) showed that elliptical galaxies can be the result of a major merger between two spiral galaxies. Subsequent numerical simulations showed that indeed various properties of elliptical galaxies and classical bulges can be recovered from simulations that use cosmological self-consistent initial orbital parameters (Khochfar \& Burkert 2006) for merging systems (see e.g. Barnes \& Hernquist 1992; Naab \& Burkert 2003; Jesseit, Naab \& Burkert 2005; Naab, Jesseit \& Burkert 2006; Jesseit et al. 2007). As a consequence it should be possible to generalise results for the formation of elliptical galaxies to the formation of classical bulges and to speak more general of the formation of spheroids (Khochfar \& Silk 2006a). E.g. it has been predicted that massive spheroids form in dry major mergers of elliptical galaxies, and that intermediate mass spheroids form as a result of a major merger between an elliptical and a spiral galaxy (Khochfar \& Burkert 2003; Naab, Khochfar \& Burkert 2006). Khochfar \& Silk (2006a) find that this is indeed the case for ellipticals as well as bulges. 
Bulges are embedded in large stellar discs in contrast to elliptical galaxies which poses the question if they really can have the same origin. The $\Lambda$ CDM paradigm offers a natural way for the transition from elliptical galaxies to bulges of early-type spirals via the accretion of a new disc in the aftermath of a major merger (Kauffmann et al. 1999; Springel \& Hernquist 2005). As Khochfar \& Burkert (2001) show the predicted merger rate of galaxies in the $\Lambda \mathrm{CDM}$ paradigm is in fair agreement with the observed one which allows to test robustly the transition in Hubble types due to the growth of a new stellar disc. Hence the properties of bulges like e.g. the isophotal shape (Khochfar \& Burkert $2005)$ will initially be set by the properties of the progenitor elliptical galaxy.

\section{Model}

We use semi-analytical modelling of galaxy formation to predict the star burst and quiescent components of elliptical galaxies. The dark matter history is calculated using the merger tree proposed by Somerville \& Kolatt (1999) with a mass resolution of $2 \times$ $10^{9} M_{\odot}$. The baryonic physics within these dark matter halos is calculated following recipes presented in Khochfar \& Burkert (2005) and Khochfar \& Silk (2006a). In our simulation, we assume that elliptical galaxies form whenever a major merger $\left(M_{1} / M_{2} \leqslant\right.$ 3.5 with $M_{1} \geqslant M_{2}$ ) takes place. We assume that during this process all the cold gas which was in the progenitor discs will be consumed in a central starburst, adding to the spheroid mass, and that all stars in the progenitor disks will be scattered into the spheroid too. Furthermore we allow the stars of satellite galaxies in minor mergers to also contribute to the spheroid. During the evolution of a galaxy, we keep track of the origins of all stars brought into the spheroid and attribute them to two categories, starburst and quiescent, where the first incorporates stars formed during a starburst in a major merger and the latter includes stars previously formed in a disc and added to the spheroid during a major merger. Each star will carry along its label and not change it, which means that if a star was made in a merger of two progenitor galaxies and the remnant of that merger participated in another merger, the star will still contribute to the merger component of the final remnant. For more modelling details, we refer the reader to Khochfar \& Silk (2006a) and references therein. Please note that our simulation does not include AGNfeedback (Schawinski et al. 2006) or environmental effects (Khochfar \& Ostriker 2007) that have influence on the most massive galaxies.

\section{Results}

Ongoing mergers constantly transfer discs stars to spheroids in the universe. If this process is more efficient than star formation in discs one is to expect an increase in the fraction of stars in spheroids over cosmic time. However, the merger rate is a strong decreasing function with redshift (Khochfar \& Burkert 2001) and at late time disc growth overtakes merging. In the left panel of figure 1 the fraction of stars in spheroids as a function of redshift and galaxy mass is shown. At early times the most massive galaxies, $M_{*}>M_{\mathrm{c}} \sim 3 \times 10^{10} \mathrm{M}_{\odot}$ are all elliptical galaxies and only at late times massive spiral galaxies appear. This is related to the gradual transformation of gas into stars in discs in contrast to the violent and fast transformation of gas into stars during major mergers. Many of the intermediate massive elliptical galaxies that formed at high redshift continue to grow discs to become bulges of present day spiral galaxies.

The right panel of figure 1 shows the quiescent fraction of bulge stars as a function of galaxy mass. The quiescent fraction increases gradually until roughly $M_{\mathrm{c}}$ where it becomes constant at $\sim 0.85$. Most of the stars in bulges therefore originated from discs 

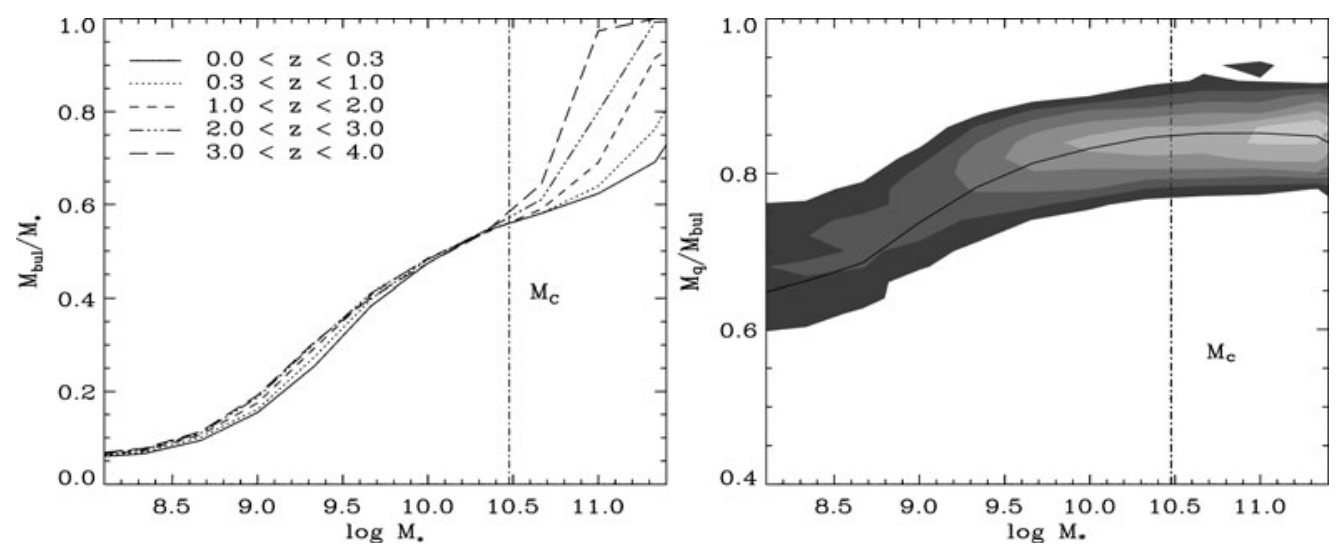

Figure 1. Left panel: Fraction of stars in galaxies of a given mass that reside in spheroids at various redshifts. Right panel: Quiescent fraction of stars in spheroids as a function of galaxy mass. The solid line shows the median of the distribution. The dot-dashed line indicates the critical mass scale $M_{\mathrm{c}}$.

of progenitor galaxies or satellite galaxies. Khochfar \& Silk (2006a) report the number of minor satellite mergers exceeds that of major mergers by an order of magnitude and is therefore one important driver for a high quiescent fraction in bulges. For massive bulges in addition mostly dry major mergers cause the quiescent fraction to stay constant and not to change much, which explains the behaviour at the high mass end.

Numerical simulations by Springel \& Hernquist (2005) show that dissipation accompanied by starbursts during major mergers leads to a population of stars that is more centrally concentrated than the scattered disc stars once they relaxed to a spheroid at the end of the merger. In our simulations we identified those centrally concentrated stars with the starburst component and the less concentrated previous disc stars with the quiescent component of bulges. Khochfar \& Silk (2006b) propose based on these two components a simple model in which the size of galaxies scales with the amount of dissipation during their formation and that is able to reproduce the size-evolution of early-type galaxies. In the left panel of figure 2 we show the expected size evolution of bulges as a function of their mass and formation time, i.e. we show the ratio of the present day effective radius of bulges, $r_{\mathrm{e}, \text { local }}$, to that of bulges at higher redshifts. Massive bulges that formed early are most likely to have had a significant amount of dissipation involved during their formation, because the gaseous disc only had enough time to transform a small portion of the gas into stars. In contrast the size-evolution for small bulges is not very strong, as there is only a small difference in the amount of dissipation.

The right panel of the same figure shows the quiescent fraction in bulges as a function of galaxy mass and environment. For galaxies more massive than $M_{\mathrm{c}}$ the quiescent fraction does not depend on the environment. Only for galaxies below $M_{\mathrm{c}}$ we find an environmental dependence which reflects itself in a larger quiescent fraction for field galaxies. The reason for this is mainly buried in the larger amount of dissipation that is involved in the formation of bulges that end up in high density environments. These galaxies form in general earlier and therefore the amount of dissipation is larger during major mergers.

Observations of core phase-space densities in spiral galaxies reveal that they are several order of magnitudes lower than those of elliptical galaxies of the same mass (Carlberg 1986). A possible solution to this problem is dissipation during starburst that can increase the phase space density in the remnant. If the centres of early-type spirals are dominated by bulges this suggest that bulges and ellipticals of the same mass must have had different 

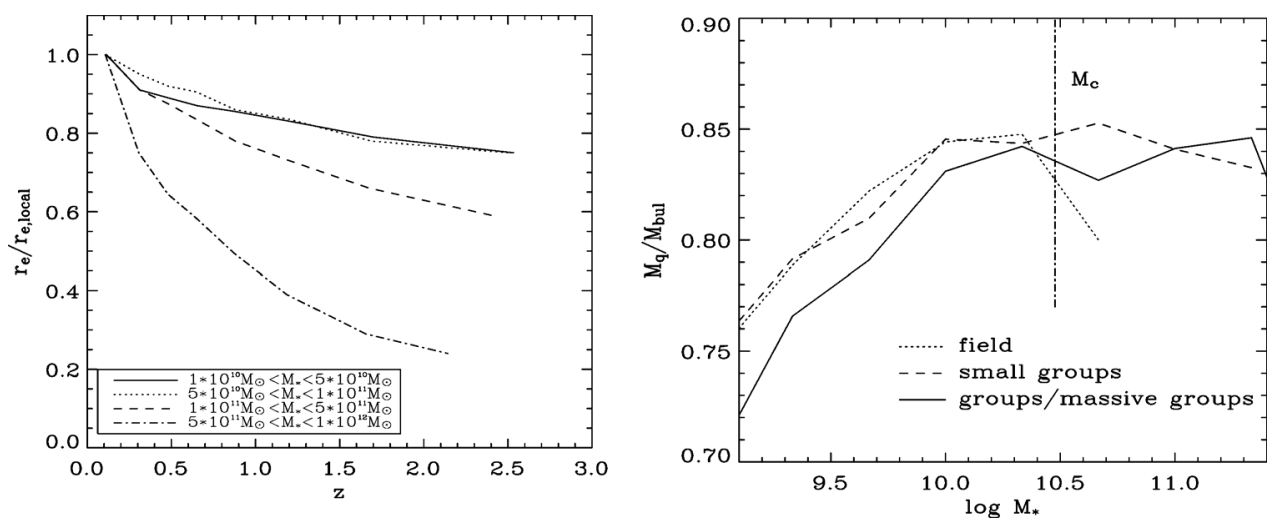

Figure 2. Left panel: size ratio between spheroids of the same mass at high redshift and locally. Right panel: quiescent fraction of stars in bulges as a function of galaxy mass and environment.

amounts of dissipation during their formation. Indeed our simulations suggest that the quiescent fraction in bulges of spiral galaxies is higher than that of ellipticals of the same mass, which could explain the observations.

\section{Conclusions}

Our results presented here indicate that the majority of stars in bulges were previously formed in discs and then later added to bulges by either major or minor mergers that occur naturally within the CDM-paradigm. Dissipation during mergers however, will be responsible for driving the size-evolution and possible environmental dependencies. This work presents a first step in differentiating stars in bulges by their origin and it will be interesting to see if signature of these two populations can indeed be observed.

\section{References}

Barnes, J. E. \& Hernquist, L. 1992, Ann. Rev. of Astronomy and Astrophysics, 30, 705

Carlberg, R. G. 1986, ApJ, 310, 593

Jesseit, R., Naab, T., \& Burkert, A. 2005, MNRAS, 360, 1185

Jesseit, R., Naab, T., Peletier, R. F., \& Burkert, A. 2007, MNRAS, 376, 997

Kauffmann, G., Colberg, J. M., Diaferio, A., \& White, S. D. M. 1999, MNRAS,303, 188

Khochfar, S. \& Burkert, A. 2001, ApJ, 561, 517

Khochfar, S. \& Burkert, A. 2003, ApJL, 597, L117

Khochfar, S. \& Burkert, A. 2005, MNRAS, 359, 1379

Khochfar, S. \& Silk, J. 2006a, MNRAS, 370, 902

Khochfar, S. \& Silk, J. 2006b, ApJL, 648, L21

Khochfar, S. \& Burkert, A. 2006, A\& A, 445, 403

Khochfar, S. \& Ostriker, J. P. 2007, ArXiv e-prints, 704, arXiv:0704.2418

Naab, T. \& Burkert, A. 2003, ApJ, 597, 893

Naab, T., Jesseit, R., \& Burkert, A. 2006, MNRAS, 372, 839

Naab, T., Khochfar, S., \& Burkert, A. 2006, ApJL, 636, L81

Sarzi, M., Rix, H. -W., Shields, J. C., Rudnick, G., Ho, L. C., McIntosh, D. H., Filippenko, A. V., \& Sargent, W. L. W. 2001, ApJ, 550, 65

Schawinski, K., et al. 2006, Nature, 442, 888

Somerville, R. S. \& Kolatt, T. S. 1999, MNRAS, 305, 1

Springel, V. \& Hernquist, L. 2005, ApJL, 622, L9

Toomre, A. \& Toomre, J. 1972, ApJ, 178, 623 